\title{
Sensory- and Motor-Related Responses of Layer 1 Neurons in the Mouse Visual Cortex
}

\author{
Lukas Mesik, ${ }^{1,3}$ Junxiang J. Huang, ${ }^{1,4}$ Li I. Zhang, ${ }^{1,2}$ and Huizhong W. Tao ${ }^{1,2}$ \\ ${ }^{1}$ Zilkha Neurogenetic Institute, ${ }^{2}$ Department of Physiology and Neuroscience, ${ }^{3}$ Neuroscience Graduate Program, and ${ }^{4}$ Graduate Program in Biological and \\ Biomedical Sciences, University of Southern California, Los Angeles, California 90033
}

Cortical layer 1 (L1) contains a sparse and molecularly distinct population of inhibitory interneurons. Their location makes them ideally suited for affecting computations involving long-range corticocortical and subcortical inputs, yet their response properties remain largely unexplored. Here we attempt to characterize some of the functional properties of these neurons in the primary visual cortex of awake mice. We find that the strongest driver of L1 neuron activity is locomotion, with at least half of L1 neurons displaying locomotionrelated activity. Visual responses are present in a similar fraction of neurons, but these responses are weaker and frequently suppressive. We also find that $\sim 43 \%$ of $\mathrm{L} 1$ neurons respond to noise stimuli and at least $14 \%$ respond to whisker touch, with these two populations being statistically independent. Finally, we find that $45 \%$ of L1 neurons have generally weak responses correlated with whisking activity. Overall, the spatial distributions of modality-specific responses were more or less random. Our work helps to establish the basic sensoryand motor-related responses of L1 interneurons, revealing several previously unreported characteristics.

Key words: awake mouse; $\mathrm{Ca}^{2+}$ imaging; functional response properties; inhibitory neuron; multimodal; visual cortex

\section{Significance Statement}

Cortical processing even in primary sensory areas is strongly influenced by nonlocal corticocortical and neuromodulatory inputs. Many of these inputs are known to converge onto layer 1 where they target not only distal dendrites of pyramidal neurons but also a sparse population of inhibitory neurons. Previous studies have suggested that layer 1 neurons may play a crucial role in mediating the effects of these long-range projections, but the different types of inputs have mostly been studied in isolation. Here, we take a closer look at the response properties of layer 1 neurons in mouse visual cortex, examining both their visual properties, likely caused by direct thalamocortical inputs, and other sensory and motor properties, likely reflecting corticocortical and neuromodulatory inputs.

\section{Introduction}

Layer 1 (L1) comprises the upper 100-120 $\mu \mathrm{m}$ of the cortex, where inputs coming from a wide range of cortical and subcortical regions converge onto the distal apical dendrites of pyramidal neurons located in deeper layers. In addition to the dense, intermingled neuropil, L1 also contains a relatively sparse population of inhibitory interneurons. Most of these interneurons are unique to L1 and belong to the larger class of 5HT3aR-expressing interneurons (Rudy et al., 2011; Lee et al., 2010). Morphologically, L1 neurons are classified into single-bouquet cells (SBCs)

Received July 18, 2019; revised 0ct. 17, 2019; accepted 0ct. 22, 2019.

Author contributions: L.I.Z. and H.W.T. designed research; L.M. and J.J.H. performed research; L.M. analyzed data; L.M., L.I.Z., and H.W.T. wrote the paper.

This work was supported by grants from the U.S. National Institutes of Health to H.W.T. (EY-019049 and EY022478) and L.I.Z. (R01-DC-008983 and RF1-MH-114112).

The authors declare no competing financial interests

Correspondence should be addressed to Huizhong W. Tao at htao@usc.edu or Li I. Zhang at liizhang@usc.edu.

https://doi.org/10.1523/JNEUROSCl.1722-19.2019

Copyright $\odot 2019$ the authors and elongated neurogliaform cells (ENGCs; Jiang et al., 2013; Lee et al., 2015). These two cell types are thought to be involved in disinhibiting pyramidal cells in the same column (center disinhibition) and inhibiting pyramidal cells across multiple columns (surround inhibition) respectively. Together, they may play a role in selecting attentional and salient signals (Jiang et al., 2013; Lee et al., 2015).

L1 neurons are uniquely positioned to be activated by longrange inputs. A diverse array of thalamocortical, corticocortical, and neuromodulatory projections reach L1 (Levitt and Moore, 1978; Zhu and Zhu, 2004; Alitto and Dan, 2013; Zhang et al., 2014; Garcia-Munoz and Arbuthnott, 2015; Ibrahim et al., 2016; Ji et al., 2016; Roth et al., 2016), sometimes extensively arborizing within the layer. The axons of local pyramidal neurons, on the other hand, rarely extend toward L1 (Burkhalter, 1989; Thomson and Lamy, 2007), suggesting that they are less likely to directly contribute to L1 interneuron activation.

It is still unknown whether all projections targeting L1 innervate $\mathrm{L} 1$ interneurons, but in all cases tested so far, direct connec- 
tions have been found. In the primary visual cortex, these include projections from the auditory cortex (Ibrahim et al., 2016), anterior cingulate cortex (Leinweber et al., 2017), thalamus (Ji et al., 2016), and basal forebrain (Alitto and Dan, 2013). L1 interneurons can therefore be activated potentially by a broad range of sensory, motor, neuromodulatory, and other higher-order inputs. However, how these disparate inputs shape L1 responses remains an open question.

Only a few studies have examined functional responses of L1 neurons in vivo. In the barrel cortex, L1 neurons have been shown to respond to whisker stimulation (Zhu and $\mathrm{Zhu}, 2004)$. The short latency of these responses suggests that they are due to direct thalamocortical inputs. In both primary auditory and primary visual cortices, L1 neurons are activated by footshocks (Letzkus et al., 2011). This activation is mediated by nicotinic currents and leads to disinhibition of pyramidal neurons, facilitating auditory fear learning. Our own previous work has shown that L1 neurons receive input from the auditory cortex and can respond to sound stimulation (Ibrahim et al., 2016). L1 activation in this circuit led to auditory sharpening of orientation selectivity in L2/3 pyramidal neurons (Ibrahim et al., 2016; McClure and Polack, 2019). Collectively, these studies demonstrate in vivo activation of L1 neurons by thalamocortical, cholinergic, and corticocortical inputs.

In the present study, our goal is to more systematically examine the conditions under which L1 neurons become activated. Our results in awake mice shed light on the range of response characteristics displayed by L1 neurons in the primary visual cortex (V1). First, we show that approximately half of L1 neurons respond to visual stimuli. The visual responses are typically weak but can sometimes become much stronger during locomotion or can conversely suppress locomotion response. Second, at least half of L1 neurons respond during locomotion. These locomotion-related responses tend to be stronger than the visual responses. Third, L1 neurons can also respond to noise ( $>40 \%)$ and whisker touch $(\sim 14 \%)$. These responses appear to be independent ( $\sim 6 \%$ overlap), suggesting that they are truly sensory in nature, rather than representing a higher-level mechanism such as alertness. Finally, we find that $\sim 45 \%$ of L1 neurons have whisking-related responses. Overall, our results show that the response characteristics of $\mathrm{L} 1$ interneurons reflect the diverse nature of corticocortical and subcortical inputs that converge onto layer 1.

\section{Materials and Methods}

Virus injections and cranial window implantation. We used GAD-cre or wild-type C57 mice of either sex, at 2-3 months of age. On the day of the surgery, mice were injected with dexamethasone $(2 \mathrm{mg} / \mathrm{kg}$, i.m.) and anesthetized with $2 \%$ isoflurane (5\% induction). After exposing the skull, we performed a $2 \times 2 \mathrm{~mm}$ square-shaped craniotomy centered at $2.5 \mathrm{~mm}$ lateral from midline and 3-3.5 mm posterior from bregma (posterior edge of the craniotomy along the lambdoid suture). An injection of 50-100 $\mathrm{nl}$ of the virus (AAV2.9-hsyn.flx.GCaMP6f/s or AAV2.9hsyn.GCaMP6f) was made into three to five places covering the posterior half of the window, at a depth of $200-300 \mu \mathrm{m}$. A custom-made cranial window composed of two pieces of cover glass was then inserted inside the craniotomy and secured by Vetbond glue. A metal headpost/recording chamber was then glued to the skull using Loctite 409 glue. The mouse was given an injection of ketoprofen $(5 \mathrm{mg} / \mathrm{kg}$, s.c.) at the end of the surgery and was also given Rimadyl containing mouse chow (BioServ) for $3 \mathrm{~d}$ following the surgery.

Calcium imaging and data processing. We started habituating the mice to head fixation on a treadmill $\sim 2$ weeks after the cranial window surgery, for at least $3 \mathrm{~d}$. A single imaging session lasted at most $3 \mathrm{~h}$, during which the mouse was allowed to freely move on the treadmill while it underwent the experimental procedures. Imaging was performed on a custom-built two-photon microscope using Deep See Insight laser (Spectra-Physics) and an $8 \mathrm{kHz}$ resonant scanner (Sutter Instrument). Images were acquired at $\sim 30$ frames/second (fps) using Scanimage 5.1 (Pologruto et al., 2003) and analyzed using custom scripts written in Matlab (MathWorks). After image alignment, regions of interest (ROIs) were manually drawn around cell bodies and a semiautomated algorithm determined the shape of the template that was then used to extract fluorescent traces. Pixels on the boundary of these ROIs served as a local neuropil estimate. For most analyses, we used a $0.5 \mathrm{~s}$ moving average of the fluorescence traces and subtracted $0.7 \times$ the neuropil estimate to correct for possible contamination by neuropil. The fluorescence baseline was estimated as the $60 \mathrm{~s}$ running 12.5 percentile of the fluorescence trace, and the fractional change in fluorescence $\mathrm{dF} / F_{0}=\left(\mathrm{F}-F_{0}\right) / F_{0}$ was calculated relative to this baseline. ROIs that had average fluorescence less than two times the neuropil average and ROIs whose baseline dropped $<1.1 \times$ the neuropil running average were excluded from the analysis.

Locomotion recording, processing, and analysis. Treadmill locomotion was recorded using a quadrature encoder (US Digital). To facilitate alignment to the imaging data, encoder timer was zeroed at a fixed time before the first trigger. The raw distance data were interpolated onto the imaging frame-time axis and then differentiated to obtain velocity. For all locomotion analyses, we used speed traces defined as a $0.5 \mathrm{~s}$ moving average of the absolute value of the velocity. The mouse was considered to be in the state of locomotion (or running) whenever its movement speed crossed the $2 \mathrm{~cm} / \mathrm{s}$ threshold, and the onset of locomotion was defined as the time-point when this threshold was first crossed within an uninterrupted $\geq 3 \mathrm{~s}$ bout of locomotion. The $2 \mathrm{~cm} / \mathrm{s}$ value was chosen based on our observation that such speed is rarely attained when the mouse is idly sitting, while it is always exceeded if the mouse starts walking or running.

In all our analyses, the locomotion (or "run") responses were calculated as the mean $\mathrm{dF} / F_{0}$ value within $3 \mathrm{~s}$ from the onset of locomotion minus the mean $\mathrm{dF} / F_{0}$ between 1.5 and $0.5 \mathrm{~s}$ before the onset of locomotion. To determine whether a neuron was locomotion responsive, these locomotion responses were compared with an equal number of quiescent "responses," which were sampled from periods when the mouse was quiescent (i.e., continuously below the locomotion threshold) and was not exposed to any stimuli.

Visual experiment and data analysis. The experiment consisted of $10-15$ repetitions of blocks, with each block containing moving sinusoidal gratings at 12 different orientations spaced $30^{\circ}$ apart together with four blank control stimuli. The gratings had a spatial frequency of 0.04 cycles $/{ }^{\circ}$ and temporal frequency of $2 \mathrm{~Hz}$. They were shown for $3 \mathrm{~s}$ followed by a $5 \mathrm{~s}$ interstimulus interval, during which the screen was gray (with luminance matching that of the grating stimuli). The "blank" stimulus consisted of the same gray screen used in between stimuli but shown for the full $8 \mathrm{~s}$ duration.

To evaluate whether the activity of a neuron was significantly affected by visual stimuli, we aligned $\mathrm{dF} / F_{0}$ traces to the onset of grating stimuli or to the onset of the blank control stimuli, with each trace spanning the time -1.5 to $+3 \mathrm{~s}$ from onset of the stimulus. Traces during which the mouse moved were removed. For the remaining traces, we calculated the change in fluorescence as the mean $\mathrm{dF} / F_{0} 3 \mathrm{~s}$ after stimulus onset minus the mean $\mathrm{dF} / F_{0}$ between 1.5 and $0.5 \mathrm{~s}$ before the stimulus. We compared the changes in $\mathrm{dF} / F_{0}$ after visual stimuli to the changes after the control (blank) stimuli using the Wilcoxon rank-sum test. Cells with a $p$ value $<0.05$ were considered to be significantly affected (excited or suppressed) by visual stimuli. The direction of the effect was inferred from the $Z$ value returned by the rank-sum test. For suppressed neurons $(Z<-2)$, we report the average minimum $\mathrm{dF} / F_{0}$ value poststimulus, while for excited neurons $(Z>2)$ we report the average maximum $\mathrm{dF} / F_{0}$ value poststimulus.

To calculate the orientation selectivity index (OSI) and direction selectivity index (DSI), we averaged $\mathrm{dF} / F_{0}$ traces for each of the different stimulus orientations. The maximum value of this average was then considered to be the response level $R$ for a given orientation. Global OSI (GOSI) and global DSI (GDSI) values were then calculated as previously 
described (Ibrahim et al., 2016). To check whether the orientation/direction tuning is significant, we used Bootstrap resampling, calculating the distribution of OSI/DSI using the same $\mathrm{dF} / F_{0}$ traces with scrambled stimulus labeling 10,000 times. The GOSI/GDSI value was deemed significant if it was $>9500(95 \%)$ of values from the scrambled distribution.

A separate visual experiment was used to evaluate interactions between visual responses and locomotion. In this experiment, mouse locomotion was continuously monitored and whenever the speed crossed a 2 $\mathrm{cm} / \mathrm{s}$ threshold, an event was triggered that had a $50 \%$ chance of leaving the screen blank and a 50\% chance of displaying a moving grating stimulus (the probability was adjusted to trend toward an even number of trials for each condition). In addition, every $20 \mathrm{~s}$ a visual stimulus was shown if an event was not triggered and the mouse was not moving. Only horizontal and vertical gratings were used in this experiment, and the responses to the two grating orientations were combined. Fluorescence changes for the three conditions were calculated as described above, and compared using the Kruskal-Wallis test followed by the post hoc Dunn's multiple-comparisons procedure. We only included imaging sessions with at least eight locomotion-only (run) events and eight locomotion + visual (vis) stimulus (runvis) events (visual-only trials were never a limiting factor). The visual events required mice to be inactive (speed $<1$ $\mathrm{cm} / \mathrm{s}$ ) between -4 and $+3 \mathrm{~s}$ from the event onset. Run and runvis events required mice to be inactive between -4 and $-1 \mathrm{~s}$ from the event onset.

In a small subset of neurons in which runvis responses were stronger than either run or vis responses alone, we determined whether the combined response was supralinear by forming a vis + run distribution consisting of all possible sums of vis and run responses, and then comparing runvis responses to this new distribution using the Wilcoxon rank-sum test.

Sound and touch experiment and data analysis. The experiment consisted of 3 min blocks that were repeated 8-12 times. The blocks contained three events spaced 1 min apart: a noise burst, a whisker touch by motor-mounted cotton swab, and a control condition in which the cotton swab was not moved all the way to the whiskers (to control for potentially audible sound made by the motor). The noise burst [ $80 \mathrm{~dB}$ sound pressure level (SPL)] was delivered by a speaker positioned $20 \mathrm{~cm}$ behind the mouse. Touch stimulus consisted of a cotton swab mounted on a stepper motor driven by an Arduino board. The motor extended the cotton swab toward the whiskers and then moved back and forth for $1.5 \mathrm{~s}$ at $2 \mathrm{~Hz}(\sim 1$-mm-long strokes), after which it retracted. The control condition involved the same back and forth movement at a position halfway to the whiskers, so that the sound evoked by the motor would match the stimulus condition. Calcium imaging data were processed as described above. After processing, we aligned the fluorescence traces to the onset of touch stimuli, touch control condition, and noise burst stimuli. As a control condition for noise burst stimuli, we used traces aligned to midpoints between any two consecutive events. The maximum $\mathrm{dF} / F_{0}$ was calculated by subtracting the average $\mathrm{dF} / F_{0}$ value between 1.5 and $0.5 \mathrm{~s}$ before the event from the maximum $\mathrm{dF} / F_{0}$ within $3 \mathrm{~s}$ after the event. Traces during which the mouse moved were not used for the analysis and a minimum of five valid noise/touch trials (without running) were required for the recording to be included in any noise/ touch analysis. The maximum $\mathrm{dF} / F_{0}$ values for stimulus and control conditions were compared using the Wilcoxon rank-sum test.

Analysis of whisking responses. All whisking-related analysis was restricted to periods with no locomotion, no sound stimulation, and no whisker stimulation. Whisking events were detected as whisker signals (extracted from the video; see below) that crossed the $2 \mathrm{SD}$ threshold. To calculate whisking-evoked activity, we subtracted the mean $\mathrm{dF} / F_{0}$ value $1.5-0.5 \mathrm{~s}$ before the whisking event from the maximum $\mathrm{dF} / F_{0}$ value within $2 \mathrm{~s}$ after the whisking event. For each whisking event, a control event was formed by applying the same analysis to a dummy event with random timing between -10 to $+10 \mathrm{~s}$ from the actual whisking event (excluding periods of locomotion, sound stimulation and whisker stimulation). The responses were then compared using the Wilcoxon signed rank test.

To check whether noise and touch responses could be explained by whisking activity, we matched each whisking event following noise/touch with five whisking events with the closest amplitude. The matched whisk- ing responses $\left(\mathrm{dF} / F_{0}\right.$ change calculated as above) were then compared with noise/touch responses using the Wilcoxon rank-sum test.

Video recording and processing. Video recordings were obtained under infrared illumination using an ELP-USB500W02M-L21 camera with infrared filter taken out. The $800 \times 600$ pixel frame movies were acquired at $30 \mathrm{fps}$ using OBS Studio. All further video processing was performed in MATLAB using custom scripts. Frames were first converted into grayscale and regions of interest were chosen covering the snout and the whisker regions. The absolute value of frame-to-frame changes in pixel values within the ROIs was then used as a proxy for whisker movements (confirmed by manual inspection). Video data were synchronized with calcium imaging data by recording an LED light driven by the trigger signal.

Depth and spatial distribution analysis. To determine whether the prevalence of a given property was depth dependent, we created a $3 \times 2$ contingency table with two response levels (responsive/unresponsive) and three depth ranges $(10-30,30-50$, and $50-80 \mu \mathrm{m}$; note that neuronal depth was estimated with $10 \mu \mathrm{m}$ precision, and we grouped neurons deeper than $50 \mu \mathrm{m}$ together because of smaller sample sizes), and then used a $\chi^{2}$ test to check whether responsiveness and depth are independent. To check whether populations of A- and B- responsive neurons (where $\mathrm{A} / \mathrm{B}$ could be vis/run/touch/noise/whisking) were randomly intermixed, we found the nearest neighbors of all A-responsive neurons and compared the prevalence of $\mathrm{A} / \mathrm{B}$ within $\mathrm{A}$ neighbors to the prevalence of A/B within the general population using Fisher's exact test.

Statistics. All results are shown as the mean \pm SE, unless indicated otherwise. For two-sample comparisons, we used the Wilcoxon ranksum test, and the minimum required sample size was five per group. For three-sample comparisons, we used the Kruskal-Wallis test followed by Dunn's multiple-comparison correction, and the minimum sample size was eight per group. To test equality of two proportions, we used the Fisher's exact test. To test the equality of three proportions, we used the $\chi^{2}$ test. All the analyses and statistical tests were performed using Matlab (MathWorks). No statistical methods were used to predetermine sample sizes, but our sample sizes were similar to those used by other groups and were always sufficient to allow $p$ values $<0.01$ using nonparametric tests.

\section{Results}

\section{Visual and locomotion-related responses in layer 1 neurons}

Our initial goal was to characterize the basic visual response properties of L1 neurons in V1. We imaged from L1 neurons of GCaMP6 virus-injected mice head fixed on a circular treadmill while being shown moving sinusoidal gratings of varying orientations (Fig. 1A). In total, we recorded from 137 neurons (from five mice) at depths ranging from 10 to $80 \mu \mathrm{m}$ below the pia (Fig. $1 B$ ). The neurons displayed a range of characteristics from ones that were visually responsive and sometimes orientation selective, through neurons that appeared to respond to some combination of visual stimulation and locomotion, to neurons that responded purely to locomotion without significant visual responses (Fig. 1C).

To determine whether a neuron was visually responsive, we compared fluorescence signal traces aligned to the onset of the grating stimuli to those aligned to the onset of randomly interspersed blank trials (Fig. 1D, left). Similarly, to determine whether a neuron was activated by locomotion, we compared fluorescence traces aligned to the onset of locomotion to those aligned to random onsets during the time that the mouse was not moving (Fig. 1D, right). In total, 64 of 123 neurons (52\%) were significantly affected by visual stimuli $(p<0.05$, Wilcoxon ranksum test), while 57 of 123 (46\%) were affected by locomotion (Fig. 1E). Among the locomotion-responsive neurons, 11\% were suppressed and $89 \%$ were activated, while among visually responsive neurons, $30 \%$ were suppressed and $70 \%$ were activated. Twenty-six percent of the neurons were responsive to both visual stimuli and locomotion, which is consistent with visual and lo- 
A
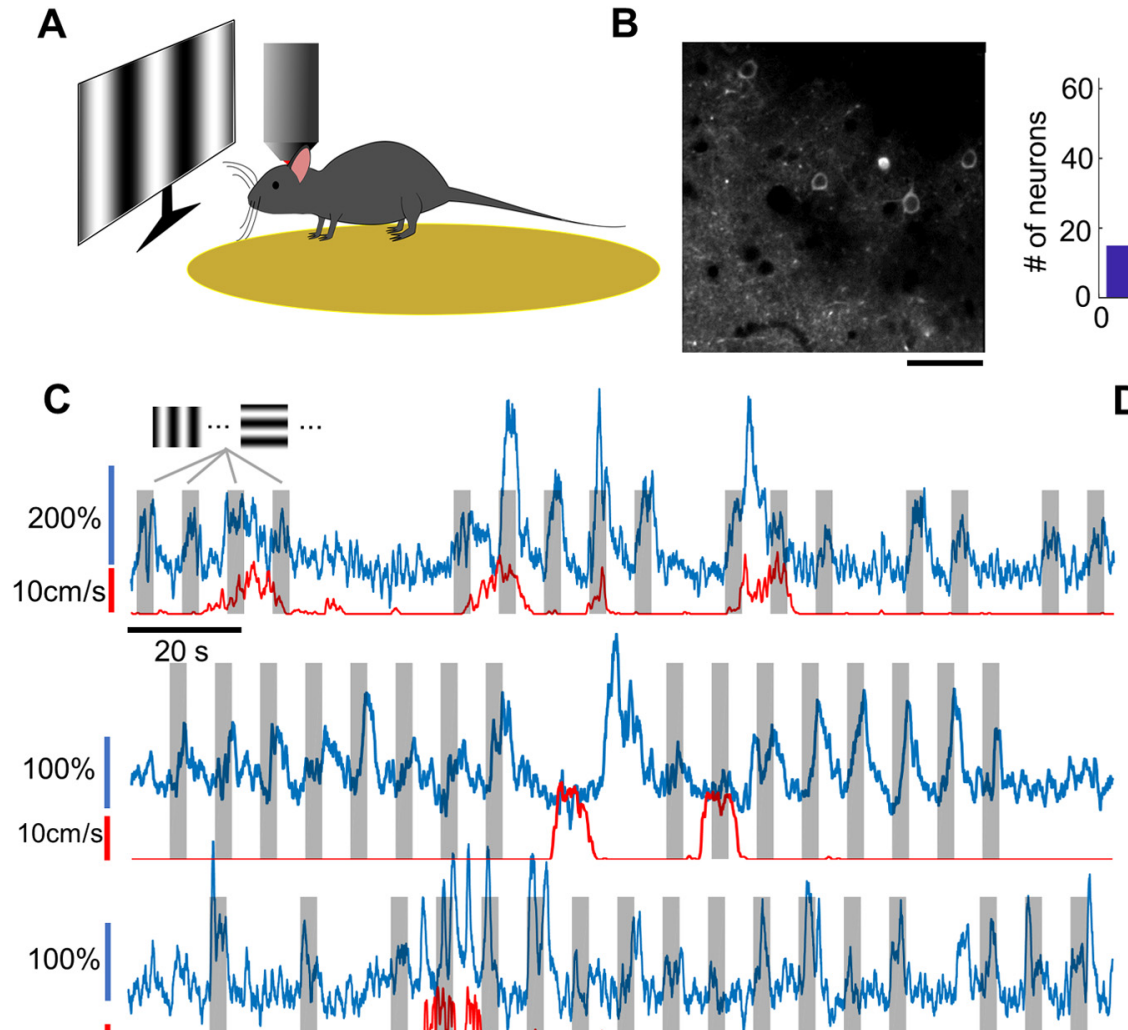
$5 \mathrm{~cm} / \mathrm{s}$

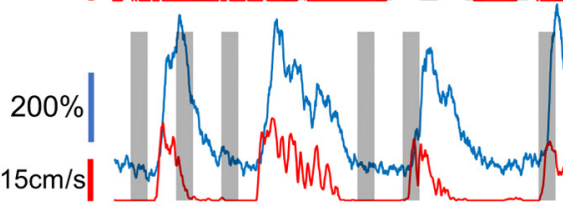

E
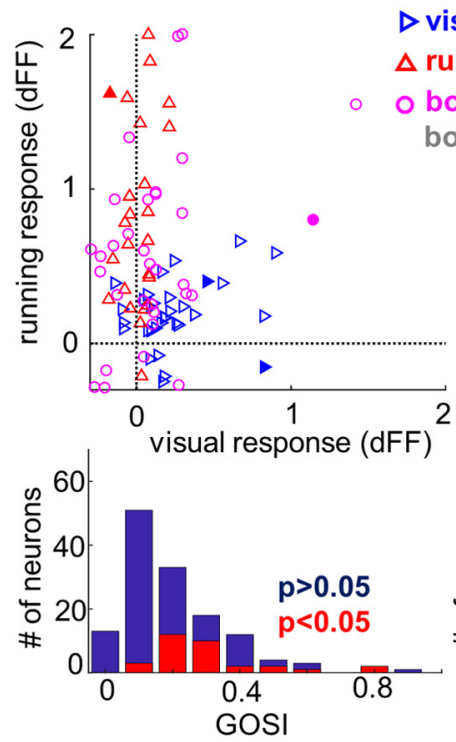

$\triangleright$ visual $p<0.05$

$\Delta$ running $\mathrm{p}<0.05$

$p<0.05$

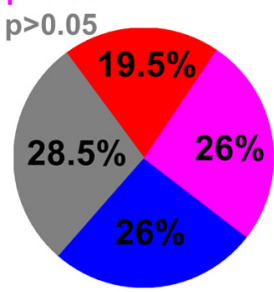

F

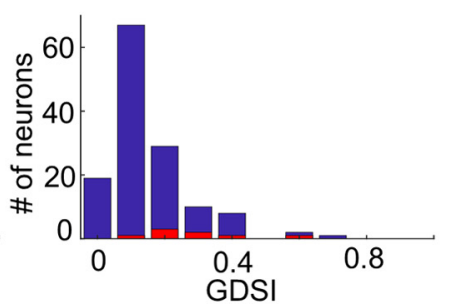

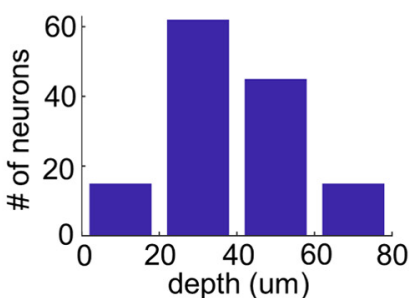

D

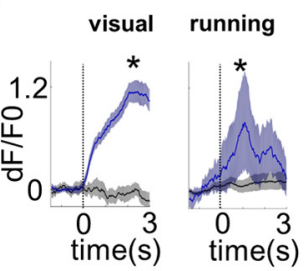

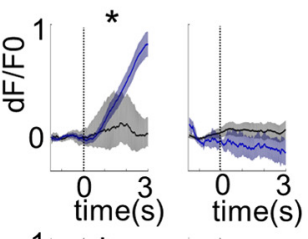
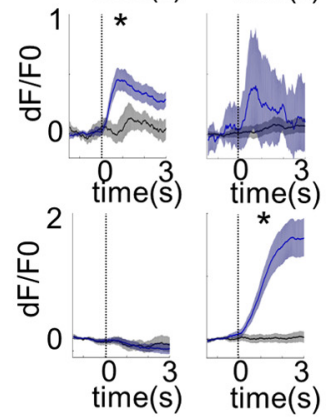

time(s)

time(s)
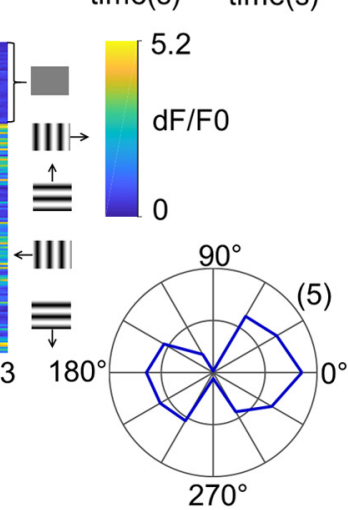

$\mathrm{GOS} \mid=0.32$ ***

GDSI $=0.1$ NS

Figure 1. Visual responsiveness of $\mathrm{L} 1$ neurons. $\boldsymbol{A}$, Schematic illustration of the experimental setup. $\boldsymbol{B}$, Left, An example imaging region. Scale bar, $50 \mu \mathrm{m}$. Right, Histogram showing the depth distribution of imaged cells, divided into four evenly spaced bins between 0 and $80 \mu \mathrm{m}$. The counts in the four bins are 15, 62, 45, and 15 cells, respectively ( $n=137$ cells across five animals). C, GCaMP6 fluorescence traces (blue, 3 min long) from four example neurons. Gray patches mark the 3-s-long visual stimulation, while the red trace below indicates the speed of locomotion. $\boldsymbol{D}$, Mean fluorescence traces (blue) aligned to the onset of visual stimulation (left) or the onset of locomotion (right). The gray trace shows the control condition, which is different for visual stimulation and running (see Materials and Methods). Shaded regions represent the $95 \%$ confidence intervals (bootstrap). ${ }^{*} p<0.05$ (rank-sum test). E, Left, Mean locomotion response versus mean visual response for significantly activated neurons. Blue triangles mark cells with significant response to visual stimuli. Red triangles mark cells with significant response to locomotion onset. Magenta circles mark cells that show significant responses under both conditions. Filled symbols correspond to the four example neurons shown in C. Right, Pie chart showing proportions of nonresponsive neurons (gray, $28.5 \%$ ), exclusively visually responsive neurons (blue, 26\%), exclusively locomotion-responsive neurons (red, 19.5\%) and neurons responding to both visual stimuli and locomotion (magenta, 26\%). $\boldsymbol{F}$, Characterization of orientation and direction selectivity for an example neuron. Left, Individual $\mathrm{dF} / F_{0}$ traces aligned to the onset of the visual stimuli. The top quarter of (Figure legend continues.) 
comotion responsiveness being independently distributed within the population ( $p=0.37$, Fisher's exact test).

Even though similar numbers of neurons were significantly activated by visual stimulation and locomotion, the responses to locomotion tended to be stronger (Fig. 1E, left). The average visually evoked response in visually responsive neurons was $\mathrm{dF} / F_{0}=0.28(\mathrm{SEM}=0.04)$, while the mean running response in locomotion-responsive neurons was three times higher, with $\mathrm{dF} / F_{0}=0.81\left(\mathrm{SEM}=0.11 ; p<10^{-8}\right.$, Wilcoxon rank-sum test; only counting positively activated neurons in both cases). Locomotion thus appears to be a more powerful driver of $\mathrm{L} 1$ responses than visual input.

To have a closer look at the visual selectivity of the neurons, we calculated their GOSI and GDSI (see Materials and Methods; Fig. $1 F$ ). The mean GOSI of $\mathrm{L} 1$ neurons was $0.21 \pm 0.16$, with $23 \%$ of neurons having statistically significant orientation selectivity (Fig. $1 G$, left). The mean GDSI was $0.16 \pm 0.14$ (Fig. $1 G$, right), but only $6 \%$ of cells was significantly direction selective, a negligible fraction given the $p=0.05$ significance level.

\section{Interactions between visual and locomotion-related responses}

Since locomotion appeared to be a major driver of L1 neurons, we wondered whether there were any interactions between visual and locomotion-related responses. Within the sinusoidal grating dataset, we encountered several cells in which visual responses appeared greatly enhanced during locomotion. However, more rigorous analysis was complicated by low sample sizes for the critical running-only (no visual stimulus) condition.

To address this problem, we designed a new stimulus set in which the onset of locomotion had a 50\% chance of triggering a visual stimulus and $50 \%$ chance of leaving the screen blank (Fig. $2 A)$. This design allowed us to obtain enough data for the three relevant conditions: visual-only (i.e., vis), running-only (i.e., run), and running and visual combined (i.e., runvis; Fig. $2 B$ ). Among the 60 cells that met our data quality and sample size criteria (see Materials and Methods), some appeared to have an enhanced response to the combination of visual stimulus with locomotion, while in some others the combined response was weaker (Fig. 2C). We had a closer look at the cells in which the runvis condition differed significantly from either the vis or run condition and found that they fell into three categories. First, in 8 of 60 neurons $(13 \%)$, runvis responses appeared stronger than either run or vis responses alone (Fig. 2D, purple and magenta). In some of these cells, including the second example in Figure $2 \mathrm{~A}$, the combined runvis response was disproportionately strong, prompting us to ask whether these responses could be supralinear. To answer this, we created the anticipated linear distribution made up of all summed pairs of run and vis responses, and compared it to the runvis distribution (see Materials and Methods). We found runvis responses in four neurons (7\%) to be significantly higher than this linear distribution (i.e., supralinear),

\footnotetext{
$\leftarrow$

(Figure legend continued.) traces correspond to the control condition with blank stimulus. The bottom three-quarters of traces are sorted by grating orientation/direction (12 in total). Right, Polar plot showing response levels of the neuron to sinusoidal gratings at different orientations. The angle corresponds to the stimulus angle, while the radius is proportional to the response level at a given orientation. For this cell, GOSI $=0.32\left(^{* * *} p<0.0001\right.$, permutation test) and GDSI $=0.1$ (NS, not significant, $p=0.11$, permutation test). $G$, Orientation (left) and direction (right) selectivity across 137 imaged neurons. Cells with significant orientation selectivity or direction selectivity $(p<0.05)$ are highlighted in red. In total, 32 of 137 cells (23.4\%) were significantly orientation selective, while only 8 of 137 cells (5.8\%) were significantly direction selective.
}

while in four of the neurons the runvis responses were consistent with being linear. The second large group of cells $(n=10$ of 60 , $17 \%$ ) were locomotion-responsive cells suppressed by visual stimuli (Fig. $2 A$, third cell for an example, $D$, blue). Finally, the third and largest group of cells ( $n=25$ of $60,42 \%)$ were ones in which the runvis and run responses were statistically identical, while they were both higher than the vis response (Fig. 2D, red). We called these cells "running-dominant" because while half of these cells were visually responsive, the combined response was not significantly affected by the visual stimulus. Notably absent were cells in which both vis and runvis responses were significantly stronger than the run response alone (see Discussion). In summary, we could detect significant running-visual interactions among $30 \%$ of $\mathrm{L} 1$ neurons, with slightly higher prevalence of suppression (Fig. $2 D$, blue) compared with facilitation (Fig. $2 D$, purple and magenta).

\section{Layer 1 responses to sound and whisker stimulation}

Our previous work has shown that L1 neurons can respond robustly to auditory stimulation (Ibrahim et al., 2016). During our recordings, we also noticed that $\mathrm{L} 1$ neurons sometimes appeared to respond to tactile stimuli, for instance when we gently applied pressure against the treadmill. We therefore sought to answer two questions. First, how common and how strong are these nonvisual sensory responses? And second, are these responses truly sensory in nature, rather than being explained by some higherlevel modulation such as alertness? We hypothesized that if the responses to sound/touch in L1 were state related, we would observe responses to whisker stimulation and auditory stimulation in the same population of neurons. If, on the other hand, the responses were sensory in nature, they would not necessarily be tied to the same population of neurons.

To address our questions, we recorded from L1 neurons in darkness while sparsely presenting either noise bursts ( $80 \mathrm{~dB}$ SPL; further referred to as "noise") or briefly stimulating the whiskers with a cotton swab driven by a motor (see Materials and Methods). As expected, we commonly observed fluorescence transients after noise bursts (Fig. 3A, top). Less commonly, we also encountered neurons with robust responses to whisker stimulation (Fig. 3A, bottom). As with visual stimuli, we aligned fluorescence changes to the onset of noise bursts or to that of whisker touch and compared these to the respective control conditions (Fig. 3B). Notably, to control for the quiet but potentially audible hum of the motor, we included control trials during which the cotton swab moved only halfway toward the whiskers (see Materials and Methods).

We found that $45 / 105$ neurons ( $42.8 \%$; from $n=5$ mice) responded to noise bursts, while only $15 / 105$ neurons (14.3\%) responded to whisker stimulation (Fig. 3C). The overlap between the two groups was 6 neurons (5.7\%), which is consistent with noise-responses and touch responses being independent within the population ( $p=1.0$, Fisher's exact test). Our results therefore clearly show that these responses do not have a common cause and are most likely sensory in nature.

Finally, we compared the strength of the noise-induced and touch-induced responses to the running-induced responses. The mean noise response across all noise-responsive neurons was $\mathrm{dF} / F_{0}=0.52(\mathrm{SEM}=0.05)$, which was approximately one-half of the mean running response of $\mathrm{dF} / F_{0}=1.1(\mathrm{SEM}=0.1$; Fig. $3 D)$. The mean touch response across all touch-responsive neurons was surprisingly high $\left(\mathrm{dF} / F_{0}=0.85, \mathrm{SEM}=0.35\right)$, but the small sample size and some large outliers make it unclear how the distribution compares to noise and running responses (Fig. $3 E$ ). 

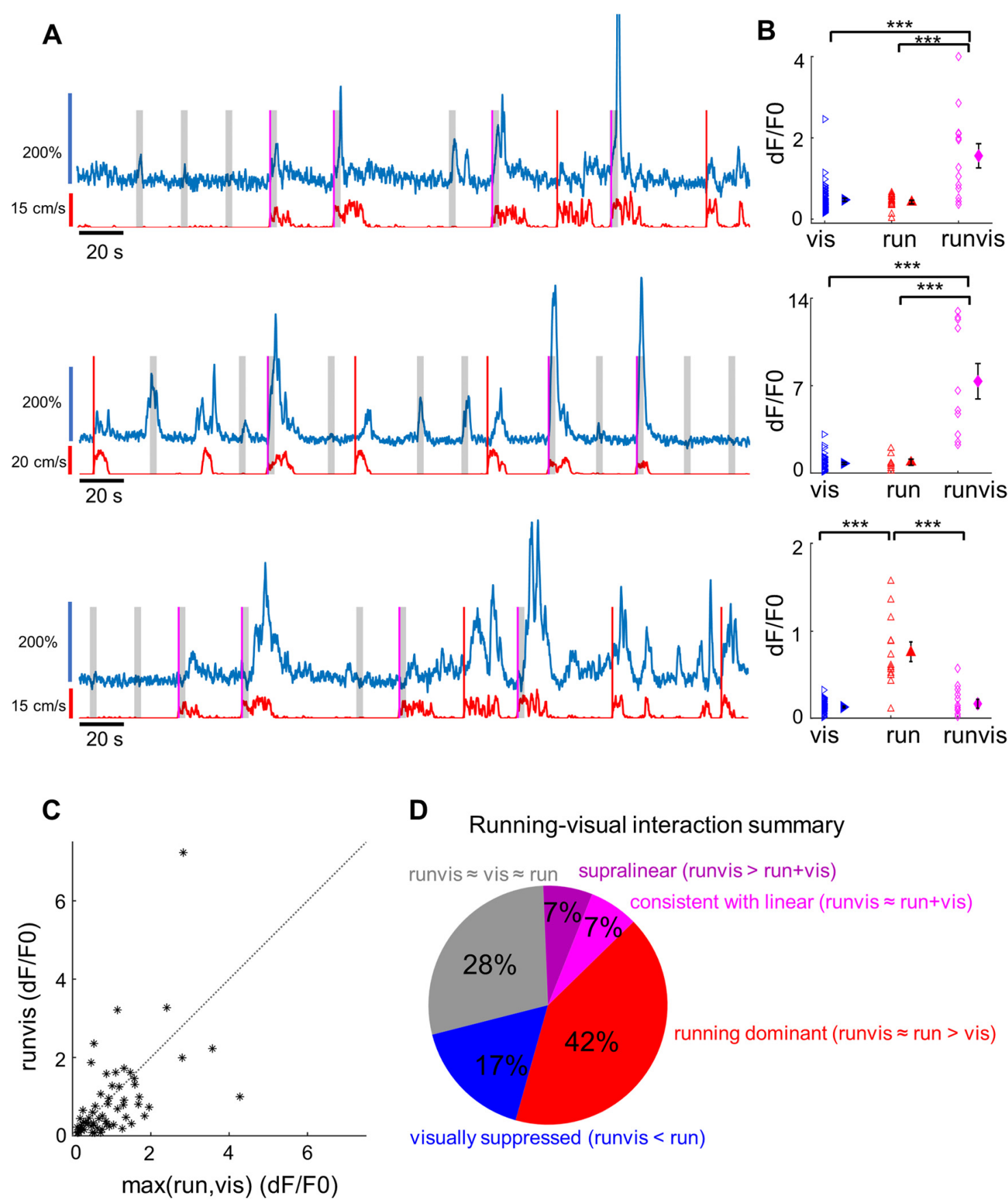

visually suppressed (runvis < run)

Figure 2. Interactions between visual and locomotion responses in L1. A, Fluorescence traces for three example L1 neurons. Red lines denote running-evoked events during which the screen remained blank, while magenta lines denote running-evoked events that triggered a 3-s-long visual stimulus (marked by the gray-shaded area). $\boldsymbol{B}$, Comparison of maximum fluorescence transients following visual stimulation (blue triangles), locomotion (red triangles), or visual stimulation during locomotion (magenta diamonds), for cells shown in $A$. In the first two neurons, the visual responses during locomotion are significantly higher than either visual responses alone or the locomotion responses alone $(p<0.0001$, Kruskal-Wallis test followed by Dunn's multiplecomparisons test). In the third neuron, responses to locomotion alone are stronger than responses to locomotion accompanied by visual stimuli ( $p<0.0001$, Kruskal-Wallis test, Dunn's multiple-comparisons test; $p<0.0001$, vis vs run; $p=0.997$, vis vs runvis; $p=0.0004$, run vs runvis). We classify such neurons as visually suppressed neurons. ${ }^{* * *}$ denotes $p<0.001$. C, The average response to visual stimuli presented during locomotion (runvis condition) plotted against the average response to the onset of running (run) or the average response to visual stimuli (vis), whichever is higher ( $n=60$ neurons across four animals). Only neurons with sufficient sample size for all three conditions ( $8+$ data points) were included in the analysis. $\boldsymbol{D}$, Pie chart summarizing running-visual interactions. The 60 cells were classified based on how visual responses during running (runvis) compared with visual-only (vis) and running-only (run) responses. In 17 of 60 cells $(28 \%$, gray), the runvis condition did not differ significantly from either the run or vis condition (runvis vs run, $p>0.05$; runvis vs vis, $p>0.05$; Kruskal-Wallis test followed by Dunn's multiple-comparisons post hoc test). In 8 of 60 cells (13.3\%, purple and magenta), runvis responses were stronger than either run or vis responses alone. In six of these cells, the runvis response was higher than both the run and vis response (runvis vs run, $p<0.05$; runvis vs vis, $p<0.05$; Kruskal-Wallis test followed by Dunn's multiple-comparisons post hoc test). In two additional cells, runvis versus run did not quite reach significance due to multiple-comparisons correction $(p<0.07)$, but was significant under ANOVA followed by Bonferroni correction, and so we decided to include these cells. The eight cells were further subdivided based on whether the runvis response was consistent with the arithmetic sum of run and vis responses (four cells, magenta, "linear"; see Materials and Methods) or whether it was significantly higher than the sum (four cells, purple, "supralinear"). In 25 of 60 cells ( $42 \%$, red), responses under the runvis condition did not differ significantly from the run condition, but both the runvis and run responses were significantly stronger than the vis response condition (runvis vs run, $p>0.05$; runvis vs vis, $p<0.05$; run vs vis, $p<0.05$; Kruskal-Wallis test followed by Dunn multiple-comparisons posthoc test). In 10 of 60 cells (17\%, blue), the runvis responses were significantly weaker than the run responses, indicating a suppressive effect of visual stimulation (runvis vs run, $p<0.05$; runvis $<$ run, Kruskal-Wallis test followed by Dunn's multiple-comparisons post hoc test). 
A

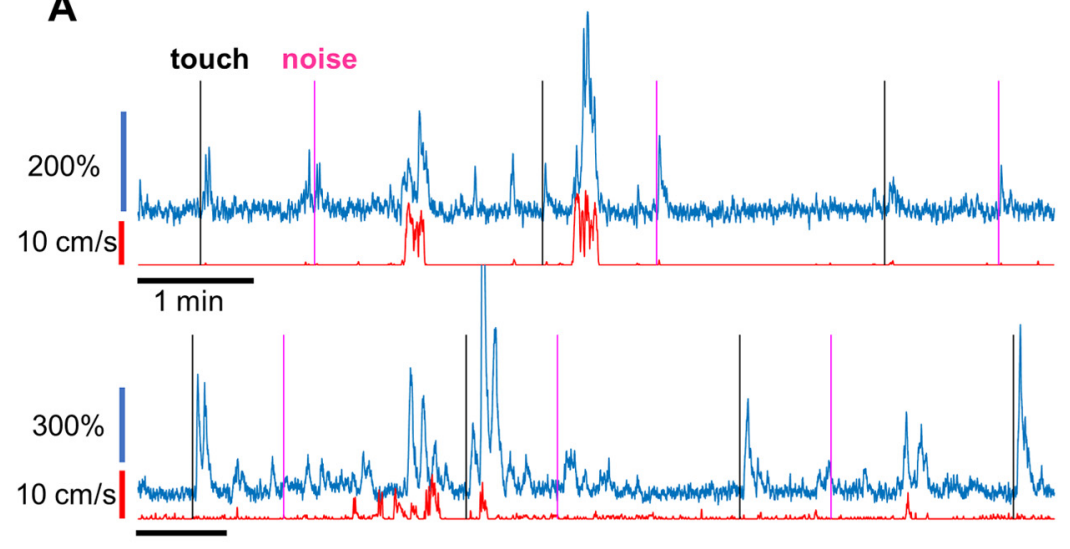

\section{C}

noise vs touch responsiveness

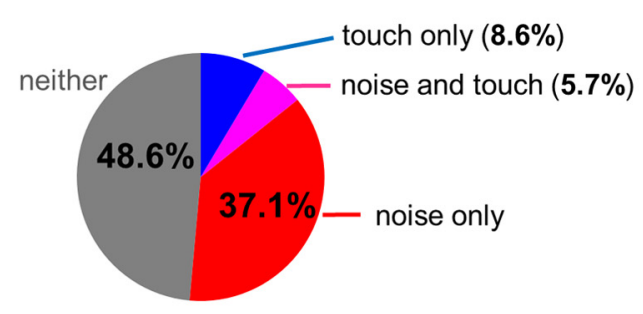

B
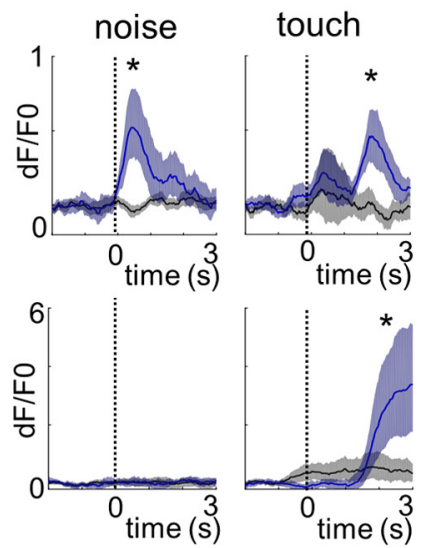

E

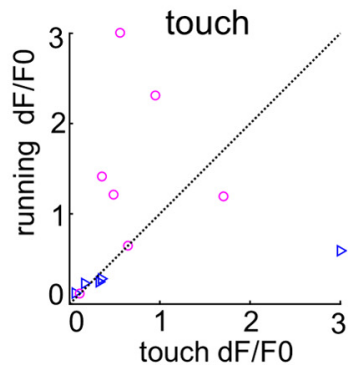

Figure 3. Noise and touch responses in L1. A, Two example L1 neurons in response to noise stimuli (marked by magenta lines) and touch stimuli (marked by black lines). $\boldsymbol{B}$, Average fluorescence traces aligned to the onset of the noise burst (left) or whisker touch (right). The black trace shows the average response in the corresponding control condition (different for noise and touch; see Materials and Methods). Shaded regions represent $95 \%$ confidence intervals (bootstrap). ${ }^{*} p<0.05$ (rank-sum test). C, Noise versus touch responsiveness summary: 39 of 105 neurons ( $37.1 \%$, shown in red) were activated only by noise, 9 of 105 neurons ( $8.6 \%$, blue) only by touch, 6 of 105 neurons (5.7\%, magenta) by both noise and touch, and 51 of 105 neurons ( $48.6 \%$, gray) by neither. $D$, Mean running response versus mean noise response scatterplot for all neurons significantly $(p<0.05)$ activated by noise. Blue triangles mark neurons that only have significant responses to noise, while magenta circles mark neurons with significant responses to both noise and locomotion. Only neurons from recordings with a sufficient level of locomotion (89 of 105 neurons) are included here. $\boldsymbol{E}$, Same as $\boldsymbol{D}$, but for touch stimuli.

\section{Whisking-related activity in layer 1 neurons}

In a subset of sound versus touch imaging sessions, we video recorded mouse whisking activity (Fig. 4A). We found that whisking was highly correlated with locomotion (Fig. $4 B$ ), so we restricted our analysis to periods of quiescence (with speed lower than the locomotion threshold). During these periods, the mouse still occasionally moved a little, and these small movements were sometimes associated with fluorescence transients. Importantly, we found that fluorescence activity was almost always better correlated with whisking than with these small movement signals (i.e., whisking-fluorescence correlation was higher than speedfluorescence correlation ( $p=1.8^{\star} 10^{-8}$, paired $t$ test; Fig. $4 C$ ), suggesting that some fluorescence transients were driven by whisking. We compared transients aligned to whisking events to the control condition (Fig. $4 D, E$ ) and found that $40 \%$ of neurons had significant whisking-related responses (Fig. 4F, blue and magenta). These responses were small on average (Fig. $4 G$, mean $\mathrm{dF} / F_{0}=0.15$ for neurons with significant responses) but could sometimes explain large transients that otherwise appeared to be random (Fig. 4D).

The presence of whisking-related activity prompted us to reexamine the noise-related responses. Indeed, noise bursts were directly followed by whisking activity in at least $84 \%$ of all the trials (Fig. 4H). To check whether the noise responses could be explained by whisking, we matched each of the noise-induced whisking events with control whisking events of similar amplitude (see Materials and Methods). Whisking could explain only noise-related transients in 6 of 41 noise-responsive neurons (Fig.
$4 I$, purple). In the rest of the neurons, noise-related responses were too large to be accounted for by whisking alone (Fig. 4I, magenta).

We performed a similar analysis in six touch-responsive neurons for which whisking data were available. Although all of them were found to be whisking responsive, whisking could explain only touch-related transients in one of these neurons. A conclusive proof that the touch responses are truly sensory in nature will require more careful experimental design, but our results so far support this interpretation.

\section{The distribution of sensory and motor responses in $\mathrm{L} 1$ is mostly random}

Throughout the study, we encountered several pairs of sensory/ motor properties that appeared to be independently distributed within the population, and so we decided to have a closer look at whether the spatial distribution of response properties is truly random. First, we checked whether the responses had any depth dependence (Fig. 5A). Of all the properties tested, only the distribution of noise responses was significantly nonrandom, with an apparent bias toward the most superficial L1 $\left(\chi^{2}(2,143)=8.01\right.$, $p=0.018$ ). However, we recorded predominantly from the top part of layer 1, so it is possible that we missed some trends that would only be apparent with larger sample sizes from a deeper part of L1. Next, to examine whether there is any evidence of functional clustering, we looked at the nearest neighbors of neurons responsive to a given modality A (A neighbors) and checked what fraction of nearest neighbors respond to this same modality 
A
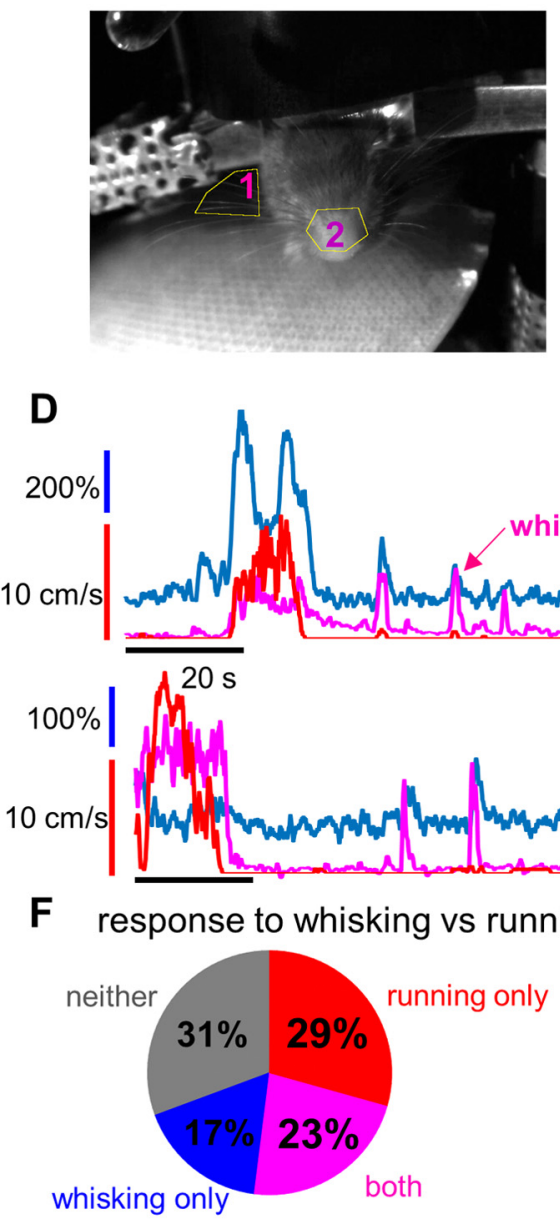

B

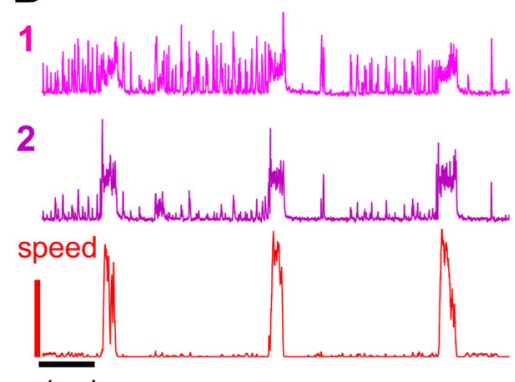

1 min
C

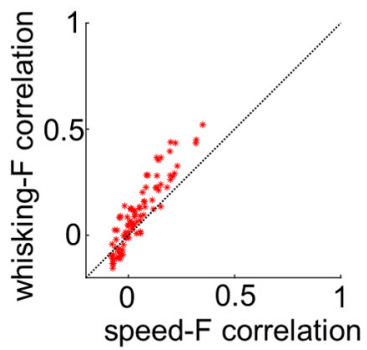

E
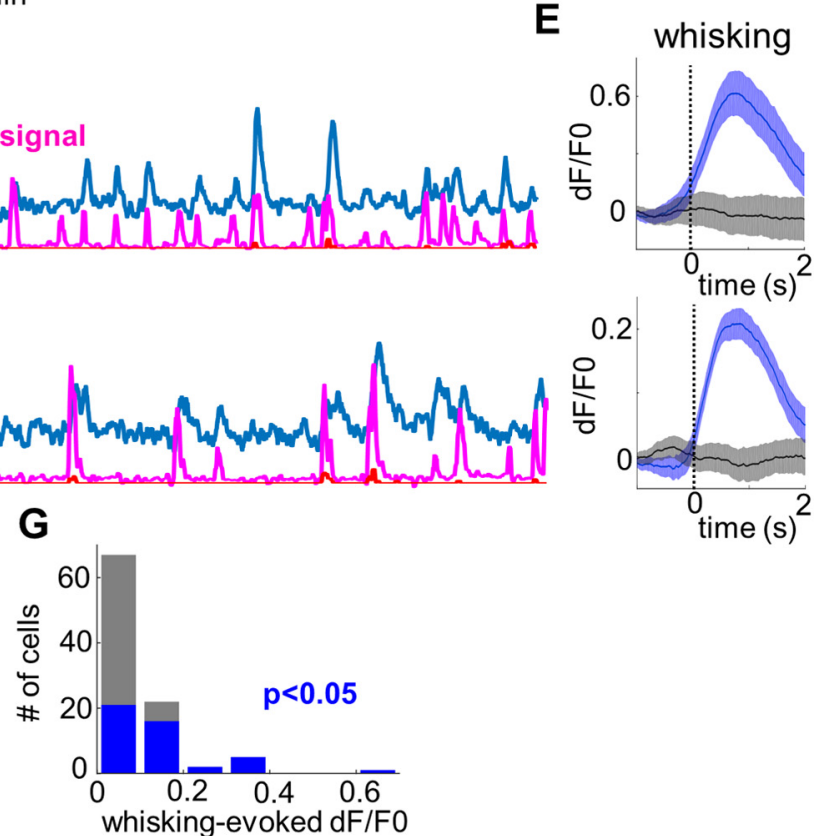

H

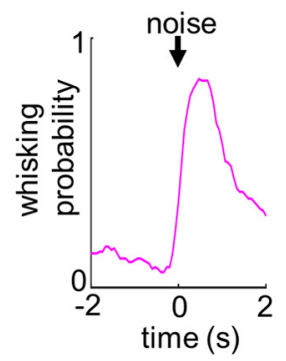

I

response to whisking vs noise

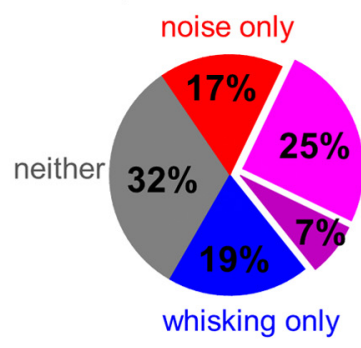

both- whisking

doesn't explain

noise response

both- whisking

can explain noise

response

Figure 4. Whisking-related activity in L1.A, Example video frame for whisking analysis, with analyzed ROls shown in yellow. $B$, Example whisking traces acquired from the two ROIs, together with the corresponding locomotion trace (bottom). $C$, Whisker- $\mathrm{dF} / F_{0}$ correlation versus speed- $\mathrm{dF} / F_{0}$ correlation analyzed during quiescent periods. Note that while many whisking bouts are accompanied by minor movements during quiescence, the correlation between fluorescence and whisking is higher than that between fluorescence and speed. $\boldsymbol{D}$, Example traces for two cells showing a high correlation between whisking activity (magenta trace) and fluorescence signal (blue). Note that some whisking-related fluorescence events happen in the complete absence of locomotion (red trace). $\boldsymbol{E}$, Average fluorescence signals aligned to whisking events (blue) or in the control condition (black) for the cells shown in $\boldsymbol{D}$. Shaded regions indicate $95 \%$ confidence intervals. $\boldsymbol{F}$, Responses to whisking versus locomotion at the population level. $0 f 75$ cells, 13 (17\%) were responsive only to whisking, 22 (29\%) only to locomotion, 17 (23\%) to both whisking and locomotion, and 23 (31\%) were not activated either during whisking or during locomotion. $\mathbf{G}$, histogram summarizing mean evoked fluorescence changes following whisking events. Cells with significant whisking responses are highlighted in blue. $\boldsymbol{H}$, Probability of whisking aligned to the onset of the noise burst. $\boldsymbol{I}$, Responses to noise versus whisking at the population level. 0 f 84 neurons, 16 (19\%) responded to whisking only, $14(17 \%)$ responded to noise only, 27 (32\%) responded to both whisking and noise, and 27 (32\%) responded to neither. Among the neurons responding both to whisking and noise, whisking could explain the noise responses in 6 of 27 cases (purple), while in the remaining 21 cases the noise responses were too high to be accounted for by whisking (magenta).

A or a second modality, B (Fig. 5B). In case of functional clustering, we would expect A neighbors to be more likely to be A responsive than the general population. On the other hand, if $A$ and $\mathrm{B}$ were randomly distributed, we would expect the fraction of Aand B-responsive neurons to be equally prevalent among A neighbors as within the overall population. Our results did not show any clear evidence of spatial clustering and instead were largely consistent with random spatial distribution (Fig. 5C-F). The only case where we saw a hint of spatial clustering was among run-responsive neurons, which were possibly overrepresented among run neighbors in both the sinusoidal moving grating dataset $(p=0.054$, Fisher's exact test comparing proportion of 
A

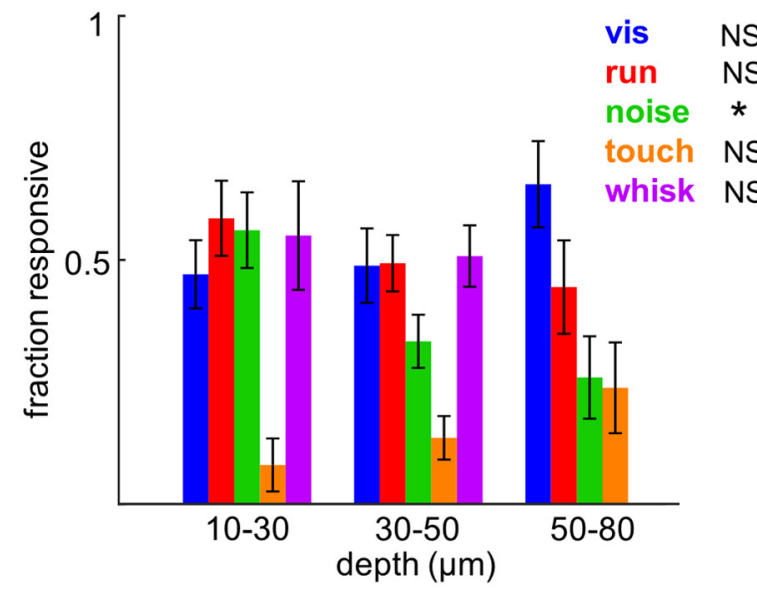

B

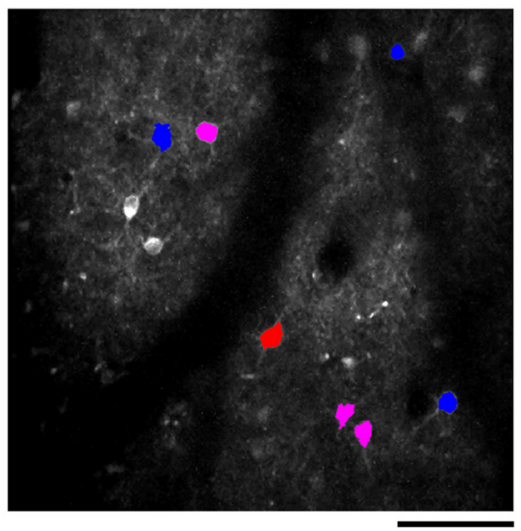
* NS NS
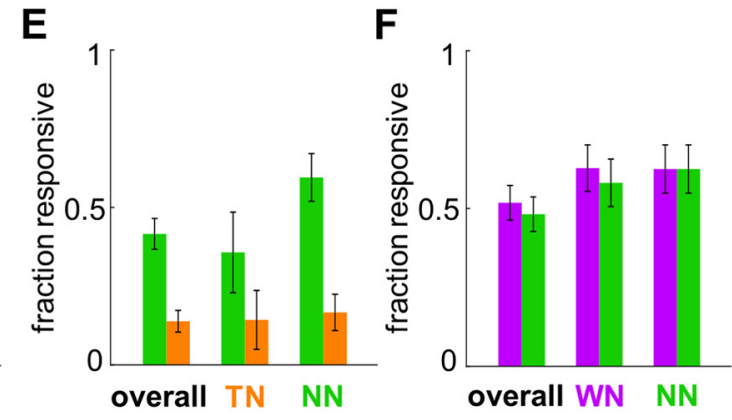

E
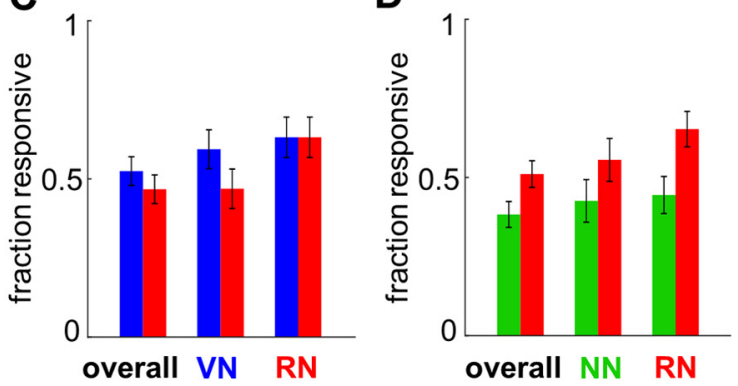

Figure 5. Depth dependence and test of spatial clustering of the sensory and motor responses in L1. A, Fraction of neurons responding to visual stimulation, running, noise, whisker touch, or whisking at different depths. Dependence of responsiveness on depth was tested using a $\chi^{2}$ test with the following results. vis(blue): $\chi^{2}(2,123)=2.79, p=0.25 ;$ run(red): $\chi^{2}(2,143)=1.48, p=$ 0.48 ; noise(green): $\chi^{2}(2,143)=8.01, p=0.018^{*}$; touch(orange): $\chi^{2}(2,105)=2.44, p=0.3$; whisk(purple): $\chi^{2}(1,84)=0.1, p=0.74$. NS, Nonsignificant. Error bars represent SE. All whisking responses were collected at depths shallower than $50 \mu \mathrm{m}$, so the prevalence of whisking responses in the $50-80 \mu \mathrm{m}$ bin is unknown. $\boldsymbol{B}$, Example image of a recorded region with neurons responding to vis, run, or both shown in blue, red, or magenta, respectively. The nearest neighbors of blue and magenta neurons are vis neighbors (VN), and the nearest neighbors of red and magenta neurons are run neighbors (RN). Scale bar, $100 \mu \mathrm{m}$. C, Fraction of vis-responsive (blue) and run-responsive (red) neurons within the overall population, among VN and among RN. The fraction of responsive neurons in the overall population was compared with the fraction of responsive neurons among vis neighbors and run neighbors using the Fisher's exact test with the following results ( $n=122$ neurons): vis among vis neighbors, $p=0.44$; run among vis neighbors, $p=1$; vis among run neighbors, $p=0.2$; run among run neighbors, $p=0.054$. $\boldsymbol{D}$, Same type of analysis as in $\boldsymbol{C}$ but looking at noise and run responsiveness among noise neighbors (NN) and RN within the noise-touch dataset ( $n=141$ neurons), as follows: noise among noise-neighbors, $p=0.63$; run among noise-neighbors, $p=0.63$; noise among run-neighbors, $p=0.43$; run among run-neighbors, $p=0.058$. $\boldsymbol{E}$, as in $\boldsymbol{C}$, but looking at noise and touch responsiveness among touch-neighbors (TN) and NN within the noise-touch dataset ( $n=101$ neurons; note that the number of neurons is different from $D$ because some recordings did not have enough valid touch trials), as follows: noise among touch-neighbors, $p=0.78$; touch among touch-neighbors, $p=1$; noise among noise-neighbors, $p=0.066$; touch among noise-neighbors, $p=0.8$. $\boldsymbol{F}$, As in $\boldsymbol{C}$ but looking at whisk and noise responsiveness among whisk-neighbors (WN) and NN within a subset of the noise-touch dataset that had video recordings ( $n=83$ neurons), as follows: whisk among whisk-neighbors, $p=0.26$; noise among whisk-neighbors, $p=0.35$; whisk among noise-neighbors, $p=0.33$; noise among noise-neighbors, $p=0.18$.

run-responsive neurons within overall population versus proportion of run-responsive neurons among run neighbors) and the noise versus touch dataset ( $p=0.058$, Fisher's exact test).

\section{Discussion}

Our study reveals a diverse array of sensory and motor responses within the L1 interneuron population. We found neurons responding to visual, auditory, and tactile stimuli, as well as neurons responding to locomotion and whisking. Responses to these different stimuli and motor events appeared to be randomly distributed, resisting our efforts to conceptually unify them. Therefore, our work suggests a picture wherein any of the many axonal projections reaching L1 can innervate any of the encountered L1 neurons in a random fashion. It is worth noting that previous studies suggest that the L1 SBCs, which contribute to center disinhibition, tend to be located in deeper L1 close to the border between L1 and L2 (Jiang et al., 2013; Lee et al., 2015). Since our imaging was mostly focused on the top part of L1, it is likely that our study has biased toward the L1 ENGCs, which contribute to surround inhibition and possibly cross-modality inhibition as well.

\section{Limited visual responsiveness of $\mathrm{L} 1$ interneurons}

In our experiments, only $\sim 50 \%$ of active L1 neurons were visually responsive, with a fraction of them being suppressed. Although the $50 \%$ figure is consistent with our previous study showing 50\% L1 innervation by geniculate axons (Ji et al., 2016), it is surprisingly low considering other sources of visual input including higher-order visual thalamus, secondary visual cortical areas, and a number of higher-order regions dealing with visual information including the retrosplenial cortex and cingulate. The most obvious explanation is that many of these neurons respond to visual stimuli we did not test, perhaps being tuned to different spatial and temporal frequencies (Glickfeld et al., 2013). However, such selectivity appears to be at odds with the poor orientation tuning we observed, comparable to that of other classes of inhibitory interneurons (Liu et al., 2009; Kerlin et al., 2010; Ma et al., 2010; Hofer et al., 2011; Kuhlman et al., 2011; Runyan and Sur, 2013; Mesik et al., 2015).

In a small subset of L1 neurons, combination of locomotion and visual stimulus evoked supralinear responses (see discussion below). In pyramidal neurons, such nonlinear responses are of- 
ten associated with burst firing (Xu et al., 2012), which has been observed in $\sim 40 \%$ of L1 SBCs (Jiang et al., 2015). The observed burst firing and supralinear responses may play a role in salience detection (Jiang et al., 2013).

\section{Locomotion as the primary driver of $\mathrm{L} 1$ activity}

Locomotion has been repeatedly shown to modulate visual responses in V1 and to directly activate multiple types of inhibitory neurons (Niell and Stryker, 2010; Polack et al., 2013; Fu et al., 2014; Mineault et al., 2016; Pakan et al., 2016). Different groups have proposed alternative sources of the locomotion related signals, including cholinergic inputs from the basal forebrain (Fu et al., 2014), noradrenergic inputs (Polack et al., 2013), and inputs from higher motor regions (Leinweber et al., 2017). In addition, axonal boutons of processes from the lateral posterior nucleus of thalamus carry locomotion-related information (Roth et al., 2016). All these inputs could potentially contribute to the locomotion-related responses in $\mathrm{L} 1$.

Layer 1 appears to be directly driven rather than simply modulated by locomotion activity. In fact, our data suggest that in the majority of L1 neurons, locomotion is the primary driver and visual input plays a modulatory role. There are several pieces of evidence pointing in this direction. First, the locomotion responses are on average three times higher than visual responses. Second, there are many exclusively locomotion-responsive neurons that clearly do not respond to visual stimuli, while the "purely" visually responsive neurons still have some locomotion responses that fail to reach significance. Third, the three groups we observed in our running-visual interaction experiments were running-dominant neurons, visually suppressed neurons, and visually facilitated neurons. We did not find any visual-dominant neurons, with similar vis and runvis responses that are both higher than the run response. These observations collectively support a model of visual input playing a modulatory rather than a driving role in the majority of L1 neurons.

\section{Consideration of $\mathrm{Ca}^{2+}$ signal as a proxy for neuronal activity}

While the use of calcium indicators for characterizing neuronal activity is widespread, certain quantitative analyses are complicated by the potential nonlinear relationship between spike number/rate and fluorescence. In particular, the finding that some neurons respond supralinearly to the runvis condition is valid only if the relationship between spike number/rate and fluorescence is linear or sublinear. For pyramidal neurons in visual cortex, the relationship appears close to linear (Chen et al., 2013), but whether this holds for L1 neurons remains to tested with in vivo simultaneous single-cell recording and $\mathrm{Ca}^{2+}$ imaging (Chen et al., 2013; Inayat et al., 2015). Another related issue is that in running-dominant neurons, the lack of activity increase during visual stimulation (runvis $\approx$ run) could be due to a saturating spike number/rate-fluorescence relationship.

\section{Independent activation by noise and touch}

The observation that $\mathrm{L} 1$ neurons can be activated by both noise and touch stimuli prompted us to ask whether such activation could have a common cause. Although we have previously demonstrated direct excitation of L1 neurons by primary auditory cortex (A1) to V1 axons (Ibrahim et al., 2016), this does not preclude the possibility that some of the sound-related responses do not originate in the auditory cortex. Indeed, as mentioned above, locomotion-related inputs do not all originate in the motor cortex either. The possibility we considered is that there is an alertness signal-possibly neuromodulatory in nature-that ac- tivates L1 neurons whenever something unexpected happens. We reasoned that if the responses to noise bursts were due to a more general alertness signal rather than sensory activation, those same neurons should also respond to a passive whisker touch. We would therefore expect a disproportionately large overlap between noise-responsive and touch-responsive neurons. Our results showed no such overlap: the noise and touch responses were statistically independent. This suggests that there is no unifying explanation for all the different responses observed in L1 and that the diversity in responses likely arises from the multitude of long-range inputs that converge onto L1.

\section{Whisker touch responses}

We observed whisker touch-related responses in 14\% of L1 neurons. These responses were recorded in darkness and were not caused by either locomotion or sound. Critically, the majority of whisker touch responses could also not be explained by whiskingrelated responses, supporting the sensory rather than motor nature of the responses. What could be the driver of these responses? There is some evidence for direct connections from the barrel cortex to V1. Monosynaptic rabies tracing from VIP neurons, which share multiple commonalities with L1 neurons, showed clear labeling in the barrel cortex (Fu et al., 2014). In addition, it has been demonstrated that stimulation of barrel cortex hyperpolarizes V1 L2/3 pyramidal neurons (Iurilli et al., 2012), an effect that might be explained by the activation of L1 neurons.

Alternatively, these responses could be mediated by one of the higher visual areas that receive direct input from the barrel cortex, or even by the temporal association area. In addition, it is possible that these responses are caused by a neuromodulatory or higher-level cognitive system, but the same system does not respond to sound stimuli, perhaps because loud sound represents a qualitatively different concern from a foreign object actively touching the whiskers.

Finally, it should be noted that the low prevalence of whisker touch responses (14\%) may be in part due to the small sample sizes involved. Since we were testing the hypothesis that noise and touch responses could both be explained by an alertness signal, the noise and whisker touch events were separated by long interstimulus intervals, which allow the mouse to relax and reduce the level of alertness. This led to small sample sizes, especially in the case of whisker touch, which required a separate control condition. Moreover, whisker touch was sometimes followed by immediate running, further decreasing sample size as such trials had to be removed. We therefore suspect that the true fraction of whisker touch-activated neurons may be higher than what we report.

\section{Whisking-related responses}

We found that a surprisingly large population of neurons ( $\sim 40 \%)$ were activated by whisking. These responses were usually weak, but could be easily detected due to large sample size (a few hundred events). The correlation was clear and sometimes hard to explain by anything other than whisking. What is the origin of this whisking-related activity? Like in the case of whisker touch, these signals could originate in the barrel cortex, which contains a population of neurons activated during whisking (de Kock and Sakmann, 2009). They could also potentially come from higher motor areas like M2, which are known to project to V1 (Fu et al., 2014; Leinweber et al., 2017). Finally, whiskingrelated activity has been observed in cholinergic axons in L1 of the barrel cortex (Eggermann et al., 2014). These axons could 
potentially also innervate $\mathrm{V} 1$ and activate $\mathrm{L} 1$ neurons through their nicotinic receptors (Alitto and Dan, 2013).

In summary, the response characteristics of the L1 interneurons we observed in this study likely reflect the diverse nature of corticocortical and subcortical inputs that converge onto layer 1.

\section{References}

Alitto HJ, Dan Y (2013) Cell-type-specific modulation of neocortical activity by basal forebrain input. Front Syst Neurosci 6:79.

Burkhalter A (1989) Intrinsic connections of rat primary visual cortex: laminar organization of axonal projections. J Comp Neurol 279:171-186.

Chen TW, Wardill TJ, Sun Y, Pulver SR, Renninger SL, Baohan A, Schreiter ER, Kerr RA, Orger MB, Jayaraman V, Looger LL, Svoboda K, Kim DS (2013) Ultrasensitive fluorescent proteins for imaging neuronal activity. Nature 499:295-300.

de Kock CP, Sakmann B (2009) Spiking in primary somatosensory cortex during natural whisking in awake head-restrained rats is cell-type specific. Proc Natl Acad Sci U S A 106:16446-16450.

Eggermann E, Kremer Y, Crochet S, Petersen CCH (2014) Cholinergic signals in mouse barrel cortex during active whisker sensing. Cell Rep 9:1654-1660.

Fu Y, Tucciarone JM, Espinosa JS, Sheng N, Darcy DP, Nicoll RA, Huang ZJ, Stryker MP (2014) A cortical circuit for gain control by behavioral state. Cell 156:1139-1152.

Garcia-Munoz M, Arbuthnott GW (2015) Basal ganglia-thalamus and the "crowning enigma." Front Neural Circuits 9:71.

Glickfeld LL, Andermann ML, Bonin V, Reid RC (2013) Cortico-cortical projections in mouse visual cortex are functionally target specific. Nat Neurosci 16:219-226.

Hofer SB, Ko H, Pichler B, Vogelstein J, Ros H, Zeng H, Lein E, Lesica NA, Mrsic-Flogel TD (2011) Differential connectivity and response dynamics of excitatory and inhibitory neurons in visual cortex. Nat Neurosci 14:1045-1052.

Ibrahim LA, Mesik L, Ji XY, Fang Q, Li HF, Li YT, Zingg B, Zhang LI, Tao HW (2016) Cross-modality sharpening of visual cortical processing through layer-1-mediated inhibition and disinhibition. Neuron 89:1031-1045.

Inayat S, Barchini J, Chen H, Feng L, Liu X, Cang J (2015) Neurons in the most superficial lamina of the mouse superior colliculus are highly selective for stimulus direction. J Neurosci 35:7992-8003.

Iurilli G, Ghezzi D, Olcese U, Lassi G, Nazzaro C, Tonini R, Tucci V, Benfenati F, Medini P (2012) Sound-driven synaptic inhibition in primary visual cortex. Neuron 73:814-828.

Jiang X, Wang G, Lee AJ, Stornetta RL, Zhu JJ (2013) The organization of two new cortical interneuronal circuits. Nat Neurosci 16:210-218.

Jiang X, Shen S, Cadwell CR, Berens P, Sinz F, Ecker AS, Patel S, Tolias AS (2015) Principles of connectivity among morphologically defined cell types in adult neocortex. Science 350:aac9462.

Ji XY, Zingg B, Mesik L, Xiao Z, Zhang LI, Tao HW (2016) Thalamocortical innervation pattern in mouse auditory and visual cortex: laminar and cell-type specificity. Cereb Cortex 26:2612-2625.

Kerlin AM, Andermann ML, Berezovskii VK, Reid RC (2010) Broadly tuned response properties of diverse inhibitory neuron subtypes in mouse visual cortex. Neuron 67:858-871.

Kuhlman SJ, Tring E, Trachtenberg JT (2011) Fast-spiking interneurons have an initial orientation bias that is lost with vision. Nat Neurosci 14:1121-1133.

Lee S, Hjerling-Leffler J, Zagha E, Fishell G, Rudy B (2010) The largest group of superficial neocortical GABAergic interneurons expresses ionotropic serotonin receptors. J Neurosci 30:16796-16808.

Lee AJ, Wang G, Jiang X, Johnson SM, Hoang ET, Lanté F, Stornetta RL, Beenhakker MP, Shen Y, Julius Zhu J (2015) Canonical organization of layer 1 neuron-led cortical inhibitory and disinhibitory interneuronal circuits. Cereb Cortex 25:2114-2126.

Leinweber M, Ward DR, Sobczak JM, Attinger A, Keller GB (2017) A sensorimotor circuit in mouse cortex for visual flow predictions. Neuron 95:1420-1432.e5.

Letzkus JJ, Wolff SB, Meyer EM, Tovote P, Courtin J, Herry C, Lüthi A (2011) A disinhibitory microcircuit for associative fear learning in the auditory cortex. Nature 480:331-335.

Levitt P, Moore RY (1978) Noradrenaline neuron innervation of the neocortex in the rat. Brain Res 139:219-231.

Liu BH, Li P, Li YT, Sun YJ, Yanagawa Y, Obata K, Zhang LI, Tao HW (2009) Visual receptive field structure of cortical inhibitory neurons revealed by two-photon imaging guided recording. J Neurosci 29:10520-10532.

Ma WP, Liu BH, Li YT, Huang ZJ, Zhang LI, Tao HW (2010) Visual representations by cortical somatostatin inhibitory neurons-selective but with weak and delayed responses. J Neurosci 30:14371-14379.

McClure JP Jr, Polack PO (2019) Pure tones modulate the representation of orientation and direction in the primary visual cortex. J Neurophysiol 121:2202-2214.

Mesik L, Ma WP, Li LY, Ibrahim LA, Huang ZJ, Zhang LI, Tao HW (2015) Functional response properties of VIP-expressing inhibitory neurons in mouse visual and auditory cortex. Front Neural Circuits 9:22.

Mineault PJ, Tring E, Trachtenberg JT, Ringach DL (2016) Enhanced spatial resolution during locomotion and heightened attention in mouse primary visual cortex. J Neurosci 36:6382-6392.

Niell CM, Stryker MP (2010) Modulation of visual responses by behavioral state in mouse visual cortex. Neuron 65:472-479.

Pakan JM, Lowe SC, Dylda E, Keemink SW, Currie SP, Coutts CA, Rochefort NL (2016) Behavioral-state modulation of inhibition is contextdependent and cell type specific in mouse visual cortex. Elife 5:e14985.

Polack PO, Friedman J, Golshani P (2013) Cellular mechanisms of brain state-dependent gain modulation in visual cortex. Nat Neurosci 16: 1331-1339.

Pologruto TA, Sabatini BL, Svoboda K (2003) ScanImage: flexible software for operating laser scanning microscopes. Biomed Eng Online 2:13.

Roth MM, Dahmen JC, Muir DR, Imhof F, Martini FJ, Hofer SB (2016) Thalamic nuclei convey diverse contextual information to layer 1 of visual cortex. Nat Neurosci 19:299-307.

Rudy B, Fishell G, Lee S, Hjerling-Leffler J (2011) Three groups of interneurons account for nearly $100 \%$ of neocortical GABAergic neurons. Dev Neurobiol 71:45-61.

Runyan CA, Sur M (2013) Response selectivity is correlated to dendritic structure in parvalbumin-expressing inhibitory neurons in visual cortex. J Neurosci 33:11724-11733.

Thomson AM, Lamy C (2007) Functional maps of neocortical local circuitry. Front Neurosci 1:19-42.

Xu NL, Harnett MT, Williams SR, Huber D, O'Connor DH, Svoboda K, Magee JC (2012) Nonlinear dendritic integration of sensory and motor input during an active sensing task. Nature 492:247-251.

Zhang S, Xu M, Kamigaki T, Hoang Do JP, Chang WC, Jenvay S, Miyamichi K, Luo L, Dan Y (2014) Long-range and local circuits for top-down modulation of visual cortex processing. Science 345:660-665.

Zhu Y, Zhu JJ (2004) Rapid arrival and integration of ascending sensory information in layer 1 nonpyramidal neurons and tuft dendrites of layer 5 pyramidal neurons of the neocortex. J Neurosci 24:1272-1279. 